\title{
@creative
commons
}

Also available at http://amc-journal.eu

ISSN 1855-3966 (printed edn.), ISSN 1855-3974 (electronic edn.)

ARS MATHEMATICA CONTEMPORANEA 8 (2015) 69-82

\section{Tight orientably-regular polytopes}

\author{
Marston Conder \\ University of Auckland \\ Auckland 1142, New Zealand \\ Gabe Cunningham \\ University of Massachusetts Boston \\ Boston, Massachusetts 02125, USA
}

Received 9 October 2013, accepted 9 February 2014, published online 7 May 2014

\begin{abstract}
It is known that every equivelar abstract polytope of type $\left\{p_{1}, \ldots, p_{n-1}\right\}$ has at least $2 p_{1} \cdots p_{n-1}$ flags. Polytopes that attain this lower bound are called tight. Here we investigate the conditions under which there is a tight orientably-regular polytope of type $\left\{p_{1}, \ldots, p_{n-1}\right\}$. We show that it is necessary and sufficient that whenever $p_{i}$ is odd, both $p_{i-1}$ and $p_{i+1}$ (when defined) are even divisors of $2 p_{i}$.
\end{abstract}

Keywords: Abstract regular polytope, equivelar polytope, flat polytope, tight polytope.

Math. Subj. Class.: 51M20, 52B15, 05E18

\section{Introduction}

Abstract polytopes are ranked partially-ordered sets that resemble the face-lattice of a convex polytope in several key ways. Many discrete geometric objects can be viewed as an abstract polytope by considering their face-lattices, but there are also many new kinds of structures that have no immediate geometric analogue.

A flag of an abstract polytope is a chain in the poset that contains one element of each rank. In many ways, it is more natural to work with the flags of a polytope rather than the faces themselves. For example, every automorphism (order-preserving bijection) of a polytope is completely determined by its effect on any single flag.

Regular polytopes are those for which the automorphism group acts transitively on the set of flags. The automorphism group of a regular polytope is a quotient of some string Coxeter group $\left[p_{1}, \ldots, p_{n-1}\right]$, and conversely, every sufficiently nice quotient of a string

E-mail addresses: m.conder@auckland.ac.nz (Marston Conder), gabriel.cunningham@gmail.com (Gabe Cunningham)

(a) (i) This work is licensed under http://creativecommons.org/licenses/by/3.0/ 
Coxeter group appears as the automorphism group of a regular polytope. Indeed, it is actually possible to reconstruct a regular polytope from its automorphism group, so that much of the study of regular polytopes is purely group-theoretic.

We say a regular polytope $\mathcal{P}$ is of type (or has Schläfli symbol) $\left\{p_{1}, \ldots, p_{n-1}\right\}$ if $\left[p_{1}, \ldots, p_{n-1}\right]$ is the minimal string Coxeter group that covers the automorphism group of $\mathcal{P}$, in a way that $p_{1}, \ldots, p_{n-1}$ are the orders of the relevant generators. There is an equivalent formulation of this property that is entirely combinatorial, and hence it is possible to define a Schläfli symbol for many non-regular polytopes, including chiral polytopes (see [10]) and other two-orbit polytopes (see [7]). Any polytope with a well-defined Schläfli symbol is said to be equivelar.

In [3], the first author determined the smallest regular polytope (by number of flags) in each rank. To begin with, he showed that every regular polytope of type $\left\{p_{1}, \ldots, p_{n-1}\right\}$ has at least $2 p_{1} \cdots p_{n-1}$ flags. Polytopes that meet this lower bound are called tight. He then exhibited a family of tight polytopes, one in each rank, of type $\{4, \ldots, 4\}$. Using properties of the automorphism groups of regular polytopes, he showed that each polytope was the smallest regular polytope in rank $n \geq 9$, and that in smaller ranks, the minimum was also attained by a tight polytope (with type or dual type $\{3\},\{3,4\},\{4,3,4\},\{3,6,3,4\}$, $\{4,3,6,3,4\},\{3,6,3,6,3,4\}$ or $\{4,3,6,3,6,3,4\}$, respectively).

The second author showed in [5] that the bound on the number of flags extended to any equivelar polytope, regardless of regularity. Accordingly, it makes sense to extend the definition of tight polytopes to include any polytope of type $\left\{p_{1}, \ldots, p_{n-1}\right\}$ with $2 p_{1} \cdots p_{n-1}$ flags. An alternate formulation was proved as well, showing that an equivelar polytope is tight if and only if every face is incident with all faces (if any) that are 2 ranks higher.

Tightness is a restrictive property, and not every Schläfli symbol is possible for a tight polytope. In order for there to be a tight polytope of type $\left\{p_{1}, \ldots, p_{n-1}\right\}$, it is necessary that no two adjacent values $p_{i}$ and $p_{i+1}$ are odd. Theorem 5.1 in [5] shows that this condition is sufficient in rank 3 . In higher ranks, the question of sufficiency is still open.

Constructing non-regular polytopes in high ranks is difficult. In order to determine which Schläfli symbols are possible for a tight polytope, it is helpful to begin by considering only regular polytopes. If every $p_{i}$ is even, then Theorem 5.3 in [3] and Theorem 6.3 in [5] show that there is a tight regular polytope of type $\left\{p_{1}, \ldots, p_{n-1}\right\}$. Also the computational data from [2,6] led the second author to conjecture that if $p$ is odd and $q>2 p$, there is no tight regular polyhedron of type $\{p, q\}$. Although we are currently unable to prove this conjecture, we can show that for tight orientably-regular polyhedra, if $p$ is odd then $q$ must divide $2 p$. Moreover, we are able to prove the following generalisation in higher ranks:

Theorem 1.1. There is a tight orientably-regular polytope of type $\left\{p_{1}, \ldots, p_{n-1}\right\}$ if and only if each of the integers $p_{i-1}$ and $p_{i+1}$ (when defined) is an even divisor of $2 p_{i}$ whenever $p_{i}$ is odd, for $1 \leq i<n$.

\section{Background}

Our background information is mostly taken from [8, Chs. 2, 3, 4], with a few small additions.

\subsection{Definition of a polytope}

Let $\mathcal{P}$ be a ranked partially-ordered set, the elements of which are called faces, and suppose that the faces of $\mathcal{P}$ range in rank from -1 to $n$. We call each face of rank $j$ a $j$-face, and we 
say that two faces are incident if they are comparable. We also call the 0 -faces, 1 -faces and $(n-1)$-faces the vertices, edges and facets of $\mathcal{P}$, respectively. A flag is a maximal chain in $\mathcal{P}$. We say that two flags are adjacent if they differ in exactly one face, and that they are $j$-adjacent if they differ only in their $j$-faces.

If $F$ and $G$ are faces of $\mathcal{P}$ such that $F \leq G$, then the section $G / F$ consists of those faces $H$ such that $F \leq H \leq G$. If $F$ is a $j$-face and $G$ is a $k$-face, then we say that the rank of the section $G / F$ is $k-j-1$. If removing $G$ and $F$ from the Hasse diagram of $G / F$ leaves us with a connected graph, then we say that $G / F$ is connected. That is, for any two faces $H$ and $H^{\prime}$ in $G / F$ (other than $F$ and $G$ themselves), there is a sequence of faces

$$
H=H_{0}, H_{1}, \ldots, H_{k}=H^{\prime}
$$

such that $F<H_{i}<G$ for $0 \leq i \leq k$ and the faces $H_{i-1}$ and $H_{i}$ are incident for $1 \leq i \leq k$. By convention, we also define all sections of rank at most 1 to be connected.

We say that $\mathcal{P}$ is an (abstract) polytope of rank $n$, or briefly, an $n$-polytope, if it satisfies the following four properties:

(a) There is a unique greatest face $F_{n}$ of rank $n$, and a unique least face $F_{-1}$ of rank -1 .

(b) Each flag has $n+2$ faces.

(c) Every section is connected.

(d) Every section of rank 1 is a diamond - that is, whenever $F$ is a $(j-1)$-face and $G$ is a $(j+1)$-face for some $j$, with $F<G$, there are exactly two $j$-faces $H$ with $F<H<G$.

Condition (d) is known as the diamond condition. Note that this condition ensures that for $0 \leq j<n$, every flag $\Phi$ has a unique $j$-adjacent flag, which we denote by $\Phi^{j}$.

In ranks $-1,0$, and 1 , there is a unique polytope up to isomorphism. Abstract polytopes of rank 2 are also called abstract polygons, and for each $2 \leq p \leq \infty$, there is a unique abstract polygon with $p$ vertices and $p$ edges, denoted by $\{p\}$.

If $F$ is a $j$-face and $G$ is a $k$-face of a polytope with $F \leq G$, then the section $G / F$ itself is a $(k-j-1)$-polytope. We may identify a face $F$ with the section $F / F_{-1}$, and call the section $F_{n} / F$ the co-face at $F$. The co-face at a vertex $F_{0}$ is also called a vertex-figure at $F_{0}$.

If $\mathcal{P}$ is an $n$-polytope, $F$ is an $(i-2)$-face of $\mathcal{P}$, and $G$ is an $(i+1)$-face of $\mathcal{P}$ with $F<G$, then the section $G / F$ is an abstract polygon. If it happens that for $1 \leq i<n$, each such section is (isomorphic to) the same polygon $\left\{p_{i}\right\}$, no matter which $(i-2)$-face $F$ and incident $(i+1)$-face $G$ we choose, then we say that $\mathcal{P}$ has Schläfli symbol $\left\{p_{1}, \ldots, p_{n-1}\right\}$, or that $\mathcal{P}$ is of type $\left\{p_{1}, \ldots, p_{n-1}\right\}$. Also when this happens, we say that $\mathcal{P}$ is equivelar.

All sections of an equivelar polytope are themselves equivelar polytopes. In particular, if $\mathcal{P}$ is an equivelar polytope of type $\left\{p_{1}, \ldots, p_{n-1}\right\}$, then all its facets are equivelar polytopes of type $\left\{p_{1}, \ldots, p_{n-2}\right\}$, and all its vertex-figures are equivelar polytopes of type $\left\{p_{2}, \ldots, p_{n-1}\right\}$.

Next, let $\mathcal{P}$ and $\mathcal{Q}$ be two polytopes of the same rank. A surjective function $\gamma: \mathcal{P} \rightarrow \mathcal{Q}$ is called a covering if it preserves incidence of faces, ranks of faces, and adjacency of flags. If there exists such a covering $\gamma: \mathcal{P} \rightarrow \mathcal{Q}$, then we say that $\mathcal{P}$ covers $\mathcal{Q}$.

The dual of a polytope $\mathcal{P}$ is the polytope obtained by reversing the partial order. If $\mathcal{P}$ is an equivelar polytope of type $\left\{p_{1}, \ldots, p_{n-1}\right\}$, then the dual of $\mathcal{P}$ is an equivelar polytope of type $\left\{p_{n-1}, \ldots, p_{1}\right\}$. 


\subsection{Regularity}

For polytopes $\mathcal{P}$ and $\mathcal{Q}$, an isomorphism from $\mathcal{P}$ to $\mathcal{Q}$ is an incidence- and rank-preserving bijection. By connectedness and the diamond condition, every polytope isomorphism is uniquely determined by its effect on a given flag. An isomorphism from $\mathcal{P}$ to itself is an automorphism of $\mathcal{P}$, and the group of all automorphisms of $\mathcal{P}$ is denoted by $\Gamma(\mathcal{P})$. We will denote the identity automorphism by $\varepsilon$.

We say that $\mathcal{P}$ is regular if the natural action of $\Gamma(\mathcal{P})$ on the flags of $\mathcal{P}$ is transitive (and hence regular, in the sense of being sharply-transitive). For convex polytopes, this definition is equivalent to any of the usual definitions of regularity.

Now let $\mathcal{P}$ be any regular polytope, and choose a flag $\Phi$, which we call a base flag. Then the automorphism group $\Gamma(\mathcal{P})$ is generated by the abstract reflections $\rho_{0}, \ldots, \rho_{n-1}$, where $\rho_{i}$ maps $\Phi$ to the unique flag $\Phi^{i}$ that is $i$-adjacent to $\Phi$. These generators satisfy $\rho_{i}^{2}=\varepsilon$ for all $i$, and $\left(\rho_{i} \rho_{j}\right)^{2}=\varepsilon$ for all $i$ and $j$ such that $|i-j| \geq 2$. Every regular polytope is equivelar, and if its Schläfli symbol is $\left\{p_{1}, \ldots, p_{n-1}\right\}$, then the order of each $\rho_{i-1} \rho_{i}$ is $p_{i}$. Note that if $\mathcal{P}$ is a regular polytope of type $\left\{p_{1}, \ldots, p_{n-1}\right\}$, then $\Gamma(\mathcal{P})$ is a quotient of the string Coxeter group $\left[p_{1}, \ldots, p_{n-1}\right]$, which is the abstract group generated by $n$ elements $x_{0}, \ldots, x_{n-1}$ subject to the defining relations $x_{i}^{2}=1,\left(x_{i-1} x_{i}\right)^{p_{i}}=1$, and $\left(x_{i} x_{j}\right)^{2}=1$ whenever $|i-j| \geq 2$.

Next, if $\Gamma$ is any group generated by elements $\rho_{0}, \ldots, \rho_{n-1}$, we define $\Gamma_{I}=\left\langle\rho_{i}\right| i \in$ $I\rangle$ for each subset $I$ of the index set $\{0,1, \ldots, n-1\}$. If $\Gamma$ is the automorphism group $\Gamma(\mathcal{P})$ of a regular polytope $\mathcal{P}$, then these subgroups satisfy the following condition, known as the intersection condition:

$$
\Gamma_{I} \cap \Gamma_{J}=\Gamma_{I \cap J} \text { for all } I, J \subseteq\{0,1, \ldots, n-1\}
$$

More generally, if $\Gamma$ is any group generated by elements $\rho_{0}, \ldots, \rho_{n-1}$ of order 2 such that $\left(\rho_{i} \rho_{j}\right)^{2}=1$ whenever $|i-j| \geq 2$, then we say that $\Gamma$ is a string group generated by involutions, and abbreviate this to say that $\Gamma$ is an sggi. If the sggi $\Gamma$ also satisfies the intersection condition (2.1) given above, then we call $\Gamma$ a string $C$-group.

There is a natural way of building a regular polytope $\mathcal{P}(\Gamma)$ from a string C-group $\Gamma$ such that $\Gamma(\mathcal{P}(\Gamma)) \cong \Gamma$ and $\mathcal{P}(\Gamma(\mathcal{P})) \cong \mathcal{P}$. In particular, the $i$-faces of $\mathcal{P}(\Gamma)$ are taken to be the cosets of the subgroup $\Gamma_{i}=\left\langle\rho_{j} \mid j \neq i\right\rangle$, with incidence of faces $\Gamma_{i} \varphi$ and $\Gamma_{j} \psi$ given by

$$
\Gamma_{i} \varphi \leq \Gamma_{j} \psi \text { if and only if } i \leq j \text { and } \Gamma_{i} \varphi \cap \Gamma_{j} \psi \neq \emptyset .
$$

This construction is also easily applied when $\Gamma$ is any sggi (not necessarily a string Cgroup), but in that case, the resulting poset is not always a polytope.

The following theory from [8] helps us determine when a given sggi is a string C-group:

Proposition 2.1. Let $\Gamma=\left\langle\rho_{0}, \ldots, \rho_{n-1}\right\rangle$ be an sggi, and $\Lambda=\left\langle\lambda_{0}, \ldots, \lambda_{n-1}\right\rangle$ a string $C$-group. If there is a homomorphism $\pi: \Gamma \rightarrow \Lambda$ sending each $\sigma_{i}$ to $\lambda_{i}$, and if $\pi$ is oneto-one on the subgroup $\left\langle\rho_{0}, \ldots, \rho_{n-2}\right\rangle$ or the subgroup $\left\langle\rho_{1}, \ldots, \rho_{n-1}\right\rangle$, then $\Gamma$ is a string C-group.

Proposition 2.2. Let $\Gamma=\left\langle\rho_{0}, \ldots, \rho_{n-1}\right\rangle$ be an sggi. If both $\left\langle\rho_{0}, \ldots, \rho_{n-2}\right\rangle$ and $\left\langle\rho_{1}, \ldots\right.$, $\left.\rho_{n-1}\right\rangle$ are string $C$-groups, and $\left\langle\rho_{0}, \ldots, \rho_{n-2}\right\rangle \cap\left\langle\rho_{1}, \ldots, \rho_{n-1}\right\rangle \subseteq\left\langle\rho_{1}, \ldots, \rho_{n-2}\right\rangle$, then $\Gamma$ is a string C-group. 
Given a regular $n$-polytope $\mathcal{P}$ with automorphism group $\Gamma=\left\langle\rho_{0}, \ldots, \rho_{n-1}\right\rangle$, we define the abstract rotations $\sigma_{1}, \ldots, \sigma_{n-1}$ by setting $\sigma_{i}=\rho_{i-1} \rho_{i}$ for $1 \leq i<n$. Then the subgroup $\left\langle\sigma_{1}, \ldots, \sigma_{n-1}\right\rangle$ of $\Gamma(\mathcal{P})$ is denoted by $\Gamma^{+}(\mathcal{P})$, and called the rotation subgroup of $\mathcal{P}$. The index of $\Gamma^{+}(\mathcal{P})$ in $\Gamma(\mathcal{P})$ is at most 2 , and when the index is exactly 2 , then we say that $\mathcal{P}$ is orientably-regular. Otherwise, if $\Gamma^{+}(\mathcal{P})=\Gamma(\mathcal{P})$, then we say that $\mathcal{P}$ is non-orientably-regular. (This notation comes from the study of regular maps.) A regular polytope $\mathcal{P}$ is orientably-regular if and only if $\Gamma(\mathcal{P})$ has a presentation in terms of the generators $\rho_{0}, \ldots, \rho_{n-1}$ such that all of the relators have even length. Note that every section of an orientably-regular polytope is itself orientably-regular.

\subsection{Flat and tight polytopes}

The theory of abstract polytopes accommodates certain degeneracies not present in the study of convex polytopes. For example, the face-poset of a convex polytope is a lattice (which means that any two elements have a unique supremum and infimum), but this need not be the case with abstract polytopes. The simplest abstract polytope that is not a lattice is the digon $\{2\}$, in which both edges are incident with both vertices. This type of degeneracy can be generalised as follows. If $\mathcal{P}$ is an $n$-polytope, and $0 \leq k<m<n$, then we say that $\mathcal{P}$ is $(k, m)$-flat if every one of its $k$-faces is incident with every one of its $m$-faces. If $\mathcal{P}$ has rank $n$ and is $(0, n-1)$-flat, then we also say simply that $\mathcal{P}$ is a flat polytope. Note that if $\mathcal{P}$ is $(k, m)$-flat, then $\mathcal{P}$ must also be $(i, j)$-flat whenever $0 \leq i \leq k<m \leq j<n$. In particular, if $\mathcal{P}$ is $(k, m)$-flat, then it is also flat.

We will also need the following, taken from [8, Lemma 4E3]:

Proposition 2.3. Let $\mathcal{P}$ be an n-polytope, and let $0 \leq k<m<i<n$. If each $i$-face of $\mathcal{P}$ is $(k, m)$-flat, then $\mathcal{P}$ is also $(k, m)$-flat. Similarly, if $0 \leq i<k<m<n$ and each co- $i$-face of $\mathcal{P}$ is $(k-i-1, m-i-1)$-flat, then $\mathcal{P}$ is $(k, m)$-flat.

It is easy to see that the converse is also true. In other words, if $\mathcal{P}$ is $(k, m)$-flat, then for $i>m$ each $i$-face of $\mathcal{P}$ is $(k, m)$-flat, and for $j<k$ each co- $j$-face of $\mathcal{P}$ is $(k-j-1, m-j-1)$-flat.

Next, we consider tightness. An equivelar polytope $\mathcal{P}$ of type $\left\{p_{1}, \ldots, p_{n-1}\right\}$ has at least $2 p_{1} \cdots p_{n-1}$ flags, by [5, Proposition 3.3]. Whenever $\mathcal{P}$ has exactly this number of flags, we say that $\mathcal{P}$ is tight. It is clear that $\mathcal{P}$ is tight if and only if its dual is tight, and that in a tight polytope, every section of rank 3 or more is tight. Also we will need the following, taken from [5, Theorem 4.4]:

Theorem 2.4. Let $n \geq 3$ and let $\mathcal{P}$ be an equivelar $n$-polytope. Then $\mathcal{P}$ is tight if and only if it is $(i, i+2)$-flat for $0 \leq i \leq n-3$.

Later in this paper we will build polytopes inductively, and for that, the following approach is useful. We say that the regular $n$-polytope $\mathcal{P}$ has the flat amalgamation property (or FAP) with respect to its $k$-faces, if adding the relations $\rho_{i}=\varepsilon$ for $i \geq k$ to $\Gamma(\mathcal{P})$ yields a presentation for $\left\langle\rho_{0}, \ldots, \rho_{k-1}\right\rangle$. Similarly, we say that $\mathcal{P}$ has the FAP with respect to its co- $k$-faces if adding the relations $\rho_{i}=\varepsilon$ for $i \leq k$ yields a presentation for $\left\langle\rho_{k+1}, \ldots, \rho_{n-1}\right\rangle$.

We will also use the following, taken from [8, Theorem 4F9]:

Theorem 2.5. Suppose $m, n \geq 2$, and $0 \leq k \leq m-2$ where $k \geq m-n$. Let $\mathcal{P}_{1}$ be a regular m-polytope, and let $\mathcal{P}_{2}$ be a regular n-polytope such that the co-k-faces of $\mathcal{P}_{1}$ are 
isomorphic to the $(m-k-1)$-faces of $\mathcal{P}_{2}$. Also suppose that $\mathcal{P}_{1}$ has the FAP with respect to its co- $k$-faces, and that $\mathcal{P}_{2}$ has the FAP with respect to its $(m-k-1)$-faces. Then there exists a regular $(k+n+1)$-polytope $\mathcal{P}$ such that $\mathcal{P}$ is $(k, m)$-flat, and the $m$-faces of $\mathcal{P}$ are isomorphic to $\mathcal{P}_{1}$, while the co- $k$-faces of $\mathcal{P}$ are isomorphic to $\mathcal{P}_{2}$. Furthermore, $\mathcal{P}$ has the FAP with respect to its $m$-faces and its co- $k$-faces.

\section{Tight orientably-regular polyhedra}

We now consider the values of $p$ and $q$ for which there is a tight orientably-regular polyhedron of type $\{p, q\}$. By [5, Proposition 3.5], there are no tight polyhedra of type $\{p, q\}$ when $p$ and $q$ are both odd. Also by [3, Theorem 5.3] and [5, Theorem 6.3], if $p$ and $q$ are both even then there exists a tight orientably-regular polyhedron whose automorphism group is the quotient of the Coxeter group $[p, q]$ obtained by adding the extra relation $\left(x_{0} x_{1} x_{2} x_{1}\right)^{2}=1$. (Note that this is the group of the polyhedron $\{p, q \mid 2\}$ described in [8, p. 196]; see [9] for more information on this and related polyhedra.) Indeed, for even $p$ and $q$, there can often be multiple (non-isomorphic) tight polyhedra; for example, there are two of type $\{4,8\}$ that are non-isomorphic (see [2]).

When $p$ is odd and $q$ is even (or vice-versa), the situation is more complicated. Evidence from [2] and [6] led the second author to conjecture that there are no tight regular polyhedra of type $\{p, q\}$ if $p$ is odd and $q>2 p$ (see [5]). We will show that this is true in the orientably-regular case. In fact, we will prove something stronger, namely that $q$ must divide $2 p$.

We start by showing that if $p$ is odd and $q$ is an even divisor of $2 p$, then there is a tight orientably-regular polyhedron of type $\{p, q\}$. To do this, we define $\Gamma(p, q)$ as the group $\left\langle\rho_{0}, \rho_{1}, \rho_{2} \mid \rho_{0}^{2}, \rho_{1}^{2}, \rho_{2}^{2},\left(\rho_{0} \rho_{1}\right)^{p},\left(\rho_{1} \rho_{2}\right)^{q},\left(\rho_{0} \rho_{2}\right)^{2},\left(\rho_{0} \rho_{1} \rho_{2} \rho_{1} \rho_{2}\right)^{2}\right\rangle$, which is obtainable by adding one extra relator to the Coxeter group $[p, q]$. (Note that this is the group of the polyhedron $\{p, q\} \cdot, 2$ described in [8, p. 196].)

Theorem 3.1. Let $p \geq 3$ be odd, and let $q$ be an even divisor of $2 p$. Then there is a tight orientably-regular polyhedron $\mathcal{P}$ of type $\{p, q\}$ such that $\Gamma(\mathcal{P}) \cong \Gamma(p, q)$.

Proof. Let $\Gamma(p, q)=\left\langle\rho_{0}, \rho_{1}, \rho_{2}\right\rangle$. In light of the construction in Section 2.2, all we need to do is show that $\Gamma(p, q)$ is a string C-group of order $2 p q$, in which the order of $\rho_{0} \rho_{1}$ is $p$ and the order of $\rho_{1} \rho_{2}$ is $q$.

First, note that the element $\omega=\left(\rho_{1} \rho_{2}\right)^{2}$ generates a cyclic normal subgroup $N$ of $\Gamma(p, q)$, since each of $\rho_{1}$ and $\rho_{2}$ conjugates $\omega$ to its inverse, and the extra relation $\left(\rho_{0} \rho_{1} \rho_{2} \rho_{1} \rho_{2}\right)^{2}=1$ implies that $\rho_{0}$ does the same. Factoring out $N$ gives quotient $\Gamma(p, 2)$, in which the extra relation $\left(\rho_{0} \rho_{1} \rho_{2} \rho_{1} \rho_{2}\right)^{2}=1$ is redundant. In fact $\Gamma(p, 2)$ is isomorphic to the string Coxeter group $[p, 2]$, which is an extension of the dihedral group of order $2 p$, and has order $4 p$.

In particular, $\Gamma(p, q)$ covers $\Gamma(p, 2) \cong[p, 2]$, and it follows that $\rho_{0} \rho_{1}$ has order $p$ (rather than some proper divisor of $p)$. Also the cover from $\Gamma(p, q)$ to $\Gamma(p, 2)$ is one-to-one on $\left\langle\rho_{0}, \rho_{1}\right\rangle$, and so by Proposition 2.1, we find that $\Gamma(p, q)$ is a string C-group.

Next, we observe that the dihedral group $D_{q}=\left\langle y_{1}, y_{2} \mid y_{1}^{2}, y_{2}^{2},\left(y_{1} y_{2}\right)^{q}\right\rangle$ is a quotient of $\Gamma(p, q)$, via an epimorphism taking $\rho_{1} \mapsto y_{1}, \rho_{2} \mapsto y_{2}$ and $\rho_{0} \mapsto\left(y_{1} y_{2}\right)^{p-1} y_{1}$. (Note that the defining relations for $\Gamma(p, q)$ are satisfied by their images in $D_{q}$, since $\left(y_{1} y_{2}\right)^{p-1} y_{1}$ has order 2 , the order of $\left(y_{1} y_{2}\right)^{p-1}$ divides $p$ (as $p-1$ is even and $q$ divides $2 p$ ), the order of $\left(y_{1} y_{2}\right)^{p-1} y_{1} y_{2}=\left(y_{1} y_{2}\right)^{p}$ divides 2 (as $q$ divides $\left.2 p\right)$, and $\left(y_{1} y_{2}\right)^{p-1} y_{2} y_{1} y_{2}$ has order 2.) In particular, the image of $\rho_{1} \rho_{2}$ is $y_{1} y_{2}$, which has order $q$, and hence $\rho_{1} \rho_{2}$ has order $q$. 
Finally, $|\Gamma(p, q)|=|\Gamma(p, q) / N||N|=|\Gamma(p, 2)||N|=4 p(q / 2)=2 p q$, since $N=$ $\left\langle\left(\rho_{1} \rho_{2}\right)^{2}\right\rangle$ has order $q / 2$.

We will show that in fact, the only tight orientably-regular polyhedra of type $\{p, q\}$ with $p$ odd are those given in Theorem 3.1. We proceed with the help of a simple lemma.

Lemma 3.2. Let $\mathcal{P}$ be an orientably-regular polyhedron of type $\{p, q\}$, with $p$ odd, and with automorphism group $\Gamma(\mathcal{P})$ generated by the reflections $\rho_{0}, \rho_{1}, \rho_{2}$. If $\omega=\left(\rho_{1} \rho_{2}\right)^{2}=\sigma_{2}^{2}$ generates a normal subgroup of $\Gamma^{+}(\mathcal{P})$, then $\omega$ is central, and q divides $2 p$.

Proof. For simplicity, let $x=\sigma_{1}=\rho_{0} \rho_{1}$ and $y=\sigma_{2}=\rho_{1} \rho_{2}$, so that $x y=\rho_{0} \rho_{2}$ and hence $x^{p}=y^{q}=(x y)^{2}=1$, and also $\omega=y^{2}$. By hypothesis, $\left\langle y^{2}\right\rangle$ is normal, and so $x y^{2} x^{-1}=$ $y^{2 k}$ for some $k$. It follows that $y^{2}=x^{p} y^{2} x^{-p}=y^{2 k^{p}}$ and that $y^{2}=(x y)^{2} y^{2}(x y)^{-2}=$ $y^{2 k^{2}}$, and therefore $2 \equiv 2 k^{2} \equiv 2 k^{p} \bmod q$. Then also $2 \equiv 2 k^{2} k^{p-2} \equiv 2 k^{p-2} \bmod q$, and by induction $2 \equiv 2 k^{p} \equiv 2 k^{p-2} \equiv \cdots \equiv 2 k \bmod q$, since $p$ is odd. Thus $x y^{2} x^{-1}=y^{2 k}=y^{2}$, and so $\omega=y^{2}$ is central. Moreover, since $y^{2}$ commutes with $x$ (which has order $p$ ) and $x y=y^{-1} x^{-1}$, we find that $y^{2 p}=x^{p} y^{2 p}=\left(x y^{2}\right)^{p}=\left(y^{-1} x^{-1} y\right)^{p}=y^{-1} x^{-p} y=1$, and so $2 p$ is a multiple of $q$.

We also utilise a connection between tight polyhedra and regular Cayley maps, as is explained in [4]. Specifically, suppose that the finite group $G$ is generated by two noninvolutory elements $x$ and $y$ such that $x y$ has order 2 , and that $G$ can be written as $A Y$ where $Y=\langle y\rangle$ is core-free in $G$ (that is, $Y$ contains no non-trivial normal subgroup of $G$ ), and $A$ is a subgroup of $G$ such that $A \cap Y=\{1\}$. Then $G$ is the group $\Gamma^{+}(M)$ of orientation-preserving automorphisms group of a regular Cayley map $M$ for the group $A$. Furthermore, this map $M$ is reflexible if and only if $G$ admits an automorphism taking $x \mapsto x y^{2}\left(=y^{-1} x^{-1} y\right)$ and $y \mapsto y^{-1}$.

Theorem 3.3. Let $p \geq 3$ be odd. If $\mathcal{P}$ is a tight orientably-regular polyhedron of type $\{p, q\}$, then $q$ is an even divisor of $2 p$, and $\Gamma(\mathcal{P})$ is isomorphic to $\Gamma(p, q)$.

Proof. Let $G=\Gamma^{+}(\mathcal{P})$, and let $\sigma_{1}=\rho_{0} \rho_{1}$ and $\sigma_{2}=\rho_{1} \rho_{2}$ be its standard generators. Also take $F=\left\langle\sigma_{1}\right\rangle$ and $V=\left\langle\sigma_{2}\right\rangle$, which are the stabilisers in $\Gamma^{+}(\mathcal{P})$ of a 2-face and incident vertex of $\mathcal{P}$. Then $F \cap V=\langle\varepsilon\rangle$ since $\mathcal{P}$ is a polytope, and $G=F V$ since $\mathcal{P}$ is tight.

Now, let $N$ be the core of $V$ in $G$ (which is the largest normal subgroup of $G$ contained in $V$ ), and let $\bar{G}=G / N, \bar{V}=V / N$ and $\bar{F}=F N / N$. Then $\bar{G}=\bar{V} \bar{F}$, and $\bar{V} \cap \bar{F}$ is trivial, and also $\bar{V}$ is core-free. Thus $\bar{G}$ is the orientation-preserving automorphism group of a regular Cayley map $M$ for the cyclic group $\bar{F}$. Furthermore, since $\mathcal{P}$ is an orientablyregular polyhedron, the group $G=\Gamma^{+}(\mathcal{P})$ has an automorphism taking $\sigma_{1} \mapsto \sigma_{1} \sigma_{2}^{2}$ and $\sigma_{2} \mapsto \sigma_{2}^{-1}$, and $\bar{G}$ has the analogous property. Hence $M$ is reflexible.

On the other hand, by [4, Theorem 3.7] we know that the only reflexible regular Cayley map for a cyclic group of odd order $p$ is the equatorial map on the sphere, with $p$ vertices of valence 2 . Thus $|\bar{V}|=2$, and so $q=|V|$ is even, and $N=\left\langle\sigma_{2}^{2}\right\rangle$.

In particular, $\left\langle\sigma_{2}^{2}\right\rangle$ is a normal subgroup of $\Gamma^{+}(\mathcal{P})$, and hence by Lemma 3.2 we also find that $q$ divides $2 p$, and that $\sigma_{2}^{2}$ is central. But $\sigma_{2}^{2}=\left(\rho_{1} \rho_{2}\right)^{2}$ is inverted under conjugation by $\rho_{1}$, and now centralised by $\sigma_{1}=\rho_{0} \rho_{1}$, and therefore also inverted under conjugation by $\rho_{0}$. Hence the relation $\left(\rho_{0} \rho_{1} \rho_{2} \rho_{1} \rho_{2}\right)^{2}=\varepsilon$ holds in $\Gamma(\mathcal{P})$, so $\Gamma(\mathcal{P})$ is a quotient of $\Gamma(p, q)$. Then finally, since $\mathcal{P}$ is tight we have $|\Gamma(\mathcal{P})|=2 p q=|\Gamma(p, q)|$, and it follows that $\Gamma(\mathcal{P}) \cong \Gamma(p, q)$. 
Combining Theorem 3.1 with Theorem 3.3 and [5, Theorem 6.3], we can now draw the following conclusion:

Theorem 3.4. There is a tight orientably-regular polyhedron of type $\{p, q\}$ if and only if one of the following is true:

(a) $p$ and $q$ are both even, or

(b) $p$ is odd and $q$ is an even divisor of $2 p$, or

(c) $q$ is odd and $p$ is an even divisor of $2 q$.

\section{Tight orientably-regular polytopes in higher ranks}

Now that we know which Schläfli symbols appear among tight orientably-regular polyhedra, we can proceed to classify the tight orientably-regular polytopes of arbitrary rank. We will say that the $(n-1)$-tuple $\left(p_{1}, \ldots, p_{n-1}\right)$ is admissible if each of $p_{i-1}$ and $p_{i+1}$ (when defined) is an even divisor of $2 p_{i}$ whenever $p_{i}$ is odd.

Theorem 4.1. If $\mathcal{P}$ is a tight orientably-regular polytope of type $\left\{p_{1}, \ldots, p_{n-1}\right\}$, then the $(n-1)$-tuple $\left(p_{1}, \ldots, p_{n-1}\right)$ is admissible.

Proof. If $\mathcal{P}$ is tight and orientably-regular, then by [5, Proposition 3.8] we know that all of its sections of rank 3 are tight and orientably-regular. Hence in particular, if $p_{i}$ is odd then the sections of $\mathcal{P}$ of type $\left\{p_{i-1}, p_{i}\right\}$ and $\left\{p_{i}, p_{i+1}\right\}$ are tight and orientably-regular. The rest now follows from Theorem 3.3.

We will prove that this necessary condition is also sufficient, which will then complete the proof of Theorem 1.1. We do this by constructing the automorphism group of a tight orientably-regular polytope of the given type.

Let $\left(p_{1}, \ldots, p_{n-1}\right)$ be an admissible $(n-1)$-tuple. Then we define the group $\Gamma\left(p_{1}, \ldots\right.$, $\left.p_{n-1}\right)$ to be the quotient of the string Coxeter group $\left[p_{1}, \ldots, p_{n-1}\right]$ obtained by adding $n-2$ extra relations $r_{1}=\cdots=r_{n-2}=1$, where

$$
r_{i}= \begin{cases}\left(x_{i-1} x_{i} x_{i+1} x_{i}\right)^{2} & \text { if } p_{i} \text { and } p_{i+1} \text { are both even, or } \\ \left(x_{i-1} x_{i} x_{i+1} x_{i} x_{i+1}\right)^{2} & \text { if } p_{i} \text { is odd and } p_{i+1} \text { is even, or } \\ \left(x_{i+1} x_{i} x_{i-1} x_{i} x_{i-1}\right)^{2} & \text { if } p_{i} \text { is even and } p_{i+1} \text { is odd. }\end{cases}
$$

Note that if $n=3$, this definition of $\Gamma\left(p_{1}, p_{2}\right)$ coincides with the one in the previous section. For $n \geq 4$, the group $\Gamma\left(p_{1}, \ldots, p_{n-1}\right)$ is the amalgamation of $\Gamma\left(p_{1}, \ldots, p_{n-2}\right)$ with $\Gamma\left(p_{2}, \ldots, p_{n-1}\right)$ in the obvious way, subject to the extra relation $\left(x_{0} x_{n-1}\right)^{2}=1$.

Also let $\mathcal{P}\left(p_{1}, \ldots, p_{n-1}\right)$ be the poset obtained from $\Gamma\left(p_{1}, \ldots, p_{n-1}\right)$, using the construction in Section 2.2. We will show that $\Gamma\left(p_{1}, \ldots, p_{n-1}\right)$ is a string C-group of order $2 p_{1} p_{2} \ldots p_{n-1}$, and then since every relator of $\Gamma\left(p_{1}, \ldots, p_{n-1}\right)$ has even length, it follows that $\mathcal{P}\left(p_{1}, \ldots, p_{n-1}\right)$ is a tight orientably-regular polytope of type $\left\{p_{1}, \ldots, p_{n-1}\right\}$.

We start by considering the order of $\Gamma\left(p_{1}, \ldots, p_{n-1}\right)$.

Proposition 4.2. Let $p_{i}$ be even. Then every element of $\Gamma\left(p_{1}, \ldots, p_{n-1}\right)$ either commutes with $\left(x_{i-1} x_{i}\right)^{2}$ or inverts it by conjugation. In particular, the square of every element of $\Gamma\left(p_{1}, \ldots, p_{n-1}\right)$ commutes with $\left(x_{i-1} x_{i}\right)^{2}$. 
Proof. Let $\omega=\left(x_{i-1} x_{i}\right)^{2}$. If $j \leq i-3$ or $j \geq i+2$, then $x_{j}$ commutes with both $x_{i-1}$ and $x_{i}$, and so commutes with $\omega$. Also it is clear that $x_{i-1}$ and $x_{i}$ both conjugate $\omega$ to $\omega^{-1}$, so it remains to consider only $x_{i-2}$ and $x_{i+1}$. Now since $p_{i}$ is even, the relator $r_{i-1}$ is either $\left(x_{i-2} x_{i-1} x_{i} x_{i-1}\right)^{2}$ or $\left(x_{i-2} x_{i-1} x_{i} x_{i-1} x_{i}\right)^{2}$. In the first case, $x_{i-2}$ commutes with $x_{i-1} x_{i} x_{i-1}$ and hence with $\left(x_{i-1} x_{i} x_{i-1}\right) x_{i}=\omega$, while in the second case, we have $\left(x_{i-2} \omega\right)^{2}=1$ and so $x_{i-2}$ conjugates $\omega$ to $\omega^{-1}$. Similarly, the relator $r_{i}$ is $\left(x_{i-1} x_{i} x_{i+1} x_{i}\right)^{2}$ or $\left(x_{i+1} x_{i} x_{i-1} x_{i} x_{i-1}\right)^{2}$, and in these two cases we find that $x_{i+1} \omega x_{i+1}=\omega$ or $\omega^{-1}$, respectively. Thus every generator $x_{j}$ of $\Gamma\left(p_{1}, \ldots, p_{n-1}\right)$ either commutes with $\left(x_{i-1} x_{i}\right)^{2}$ or inverts it by conjugation, and it follows that the same is true for every element of $\Gamma\left(p_{1}, \ldots, p_{n-1}\right)$. The rest follows easily.

Proposition 4.3. Let $\left(p_{1}, \ldots, p_{n-1}\right)$ be an admissible $(n-1)$-tuple with the property that for every $i$ strictly between 1 and $n-1$, either $p_{i}=2$ or $p_{i-1}=p_{i+1}=2$. Then $y_{i}=x_{i-1} x_{i}$ has order $p_{i}$ for all $i$, and $\left|\Gamma\left(p_{1}, \ldots, p_{n-1}\right)\right|=2 p_{1} \cdots p_{n-1}$.

Proof. We use induction on $n$, together with the observation that if $p_{j}=2$, then $1=$ $\left(x_{j-1} x_{j}\right)^{2}$, so that $x_{j-1}$ commutes with $x_{j}$, and therefore $\left\langle x_{0}, \ldots, x_{j-1}\right\rangle$ centralises $\left\langle x_{j}, \ldots, x_{n-1}\right\rangle$. First, if $p_{1}=2$, then $\Gamma\left(p_{1}, p_{2}, \ldots, p_{n-1}\right)=\Gamma\left(2, p_{2}, \ldots, p_{n-1}\right) \cong$ $\left\langle x_{0}\right\rangle \times \Gamma\left(p_{2}, \ldots, p_{n-1}\right)$, and so $\left|\Gamma\left(p_{1}, \ldots, p_{n-1}\right)\right|=2\left|\Gamma\left(p_{2}, \ldots, p_{n-1}\right)\right|=4 p_{2} \cdots p_{n-1}=$ $2 p_{1} p_{2} \cdots p_{n-1}$. Otherwise $p_{2}=2$ and $\Gamma\left(p_{1}, p_{2}, \ldots, p_{n-1}\right)=\Gamma\left(p_{1}, 2, p_{3}, \ldots, p_{n-1}\right) \cong$ $\Gamma\left(p_{1}\right) \times \Gamma\left(p_{3}, \ldots, p_{n-1}\right)$, and therefore $\left|\Gamma\left(p_{1}, \ldots, p_{n-1}\right)\right|=\left|\Gamma\left(p_{1}\right)\right|\left|\Gamma\left(p_{3}, \ldots, p_{n-1}\right)\right|=$ $2 p_{1} 2 p_{3} \cdots p_{n-1}=2 p_{1} p_{2} \cdots p_{n-1}$. The claim about the orders of the elements $y_{i}=$ $x_{i-1} x_{i}$ follows easily by induction as well.

Lemma 4.4. Let $q_{i}$ be the order of $x_{i-1} x_{i}$ in $\Gamma\left(p_{1}, \ldots, p_{n-1}\right)$, for $1 \leq i<n$. Then $q_{i}=p_{i}$ whenever $p_{i}$ is odd, and also $\left|\Gamma\left(p_{1}, \ldots, p_{n-1}\right)\right|=2 q_{1} \cdots q_{n-1}$, which divides $2 p_{1} \cdots p_{n-1}$.

Proof. Let $k_{i}=p_{i}$ when $p_{i}$ is odd, or 2 when $p_{i}$ is even. Then since $k_{i}$ divides $p_{i}$ for all $i$, there exists an epimorphism $\pi: \Gamma\left(p_{1}, \ldots, p_{n-1}\right) \rightarrow \Gamma\left(k_{1}, \ldots, k_{n-1}\right)$. Also the $(n-1)-$ tuple $\left(k_{1}, \ldots, k_{n-1}\right)$ is admissible, and indeed $k_{i-1}$ and $k_{i+1}$ are both 2 whenever $k_{i}$ is odd (since $p_{i-1}$ and $p_{i+1}$ are both even whenever $p_{i}$ is odd). Thus $\left(k_{1}, \ldots, k_{n-1}\right)$ satisfies the hypotheses of Proposition 4.3, and so $\left|\Gamma\left(k_{1}, \ldots, k_{n-1}\right)\right|=2 k_{1} \cdots k_{n-1}$.

Moreover, Proposition 4.3 tells us that when $p_{i}$ is odd, the order of the image of $x_{i}$ in $\Gamma\left(k_{1}, \ldots, k_{n-1}\right)$ is $k_{i}=p_{i}$, and so $q_{i}=p_{i}$; on the other hand, if $p_{i}$ is even, then the order of the image of $x_{i}$ in $\Gamma\left(k_{1}, \ldots, k_{n-1}\right)$ is $k_{i}=2$, and so $q_{i}$ is even in that case.

Now the kernel of the epimorphism $\pi$ is the smallest normal subgroup of $\Gamma\left(p_{1}, \ldots\right.$, $\left.p_{n-1}\right)$ containing the elements $\left(x_{i-1} x_{i}\right)^{2}$ for those $i$ such that $p_{i}$ is even. By Proposition 4.2, however, the subgroup $N$ generated by these elements is normal in $\Gamma\left(p_{1}, \ldots\right.$, $\left.p_{n-1}\right)$, and abelian. Hence in particular, $N=\operatorname{ker} \pi$, and also by the intersection condition, $|N|$ is the product of the numbers $q_{i} / 2$ over all $i$ for which $p_{i}$ is even. Thus $\left|\Gamma\left(p_{1}, \ldots, p_{n-1}\right)\right|=2 q_{1} \cdots q_{n-1}$.

In order to use Theorem 2.5 to build our tight regular polytopes recursively, we need two more observations. The first concerns the flat amalgamation property (FAP):

Proposition 4.5. If $p_{2}$ is even, then $\mathcal{P}\left(p_{1}, \ldots, p_{n-1}\right)$ has the FAP with respect to its 2-faces, and if $p_{n-2}$ is even, then $\mathcal{P}\left(p_{1}, \ldots, p_{n-1}\right)$ has the FAP with respect to its co- $(n-3)$-faces. 
Proof. Let $p_{2}$ be even, and consider the effect of killing the generators $x_{i}$ of $\Gamma\left(p_{1}, \ldots\right.$, $\left.p_{n-1}\right)$, for $i \geq 2$ (that is, by adding the relations $x_{i}=1$ to the presentation for $\Gamma\left(p_{1}, \ldots\right.$, $\left.p_{n-1}\right)$ ). Each of the relators $r_{3}, \ldots, r_{n-2}$ contains only generators $x_{i}$ with $i \geq 2$, so becomes redundant, and may be removed. The relator $r_{2}$ reduces to $x_{1}^{2}$ or $x_{1}^{4}$, while $r_{1}$ reduces to $\left(x_{0} x_{1}^{2}\right)^{2}$, which is equivalent to $x_{0}^{2}$, and hence all of these become redundant too. Thus adding the relations $x_{i}=1$ to $\Gamma\left(p_{1}, \ldots, p_{n-1}\right)$ has the same effect as adding the relations $x_{i}=1$ to the string Coxeter group $\left[p_{1}, \ldots, p_{n-1}\right]$. It is easy to see that this gives the quotient group with presentation $\left\langle x_{0}, x_{1} \mid x_{0}^{2}, x_{1}^{2},\left(x_{0} x_{1}\right)^{p_{1}}\right\rangle$, which is the automorphism group of the 2 -faces of $\mathcal{P}\left(p_{1}, \ldots, p_{n-1}\right)$. Thus $\mathcal{P}\left(p_{1}, \ldots, p_{n-1}\right)$ has the FAP with respect to its 2 -faces. The second claim can be proved by a dual argument.

Proposition 4.6. Let $\mathcal{P}$ be an equivelar n-polytope with tight $m$-faces and tight co-k-faces, where $m \geq k+3$. Then $\mathcal{P}$ is tight.

Proof. Since the $m$-faces are tight, they are $(i, i+2)$-flat for $0 \leq i \leq m-3$, by Theorem 2.4, and then by Proposition 2.3, the polytope $\mathcal{P}$ is $(i, i+2)$-flat for $0 \leq i \leq m-3$. Similarly, the co- $k$-faces are $(i, i+2)$-flat for $0 \leq i \leq n-k-4$, and $\mathcal{P}$ is $(i, i+2)$-flat for $k+1 \leq i \leq n-3$. Finally, since $m \geq k+3$, we see that $\mathcal{P}$ is $(i, i+2)$-flat for $0 \leq i \leq n-3$, and again Theorem 2.4 applies, to show that $\mathcal{P}$ is tight.

We can now prove the following.

Theorem 4.7. Let $\left(p_{1}, \ldots, p_{n-1}\right)$ be an admissible $(n-1)$-tuple, with $n \geq 4$. Also suppose that $p_{i-1}$ and $p_{i+1}$ are both even, for some $i$ (with $\left.2 \leq i \leq n-2\right)$. If $\mathcal{P}\left(p_{1}, \ldots, p_{i}\right)$ is a tight orientably-regular polytope of type $\left\{p_{1}, \ldots, p_{i}\right\}$, and $\mathcal{P}\left(p_{i}, \ldots, p_{n-1}\right)$ is a tight orientablyregular polytope of type $\left\{p_{i}, \ldots, p_{n-1}\right\}$, then $\mathcal{P}\left(p_{1}, \ldots, p_{n-1}\right)$ is a tight orientably-regular polytope of type $\left\{p_{1}, \ldots, p_{n-1}\right\}$.

Proof. Let $\mathcal{P}_{1}=\mathcal{P}\left(p_{1}, \ldots, p_{i}\right)$ and $\mathcal{P}_{2}=\mathcal{P}\left(p_{i}, \ldots, p_{n-1}\right)$, which by hypothesis are tight orientably-regular polytopes of the appropriate types. Since $p_{i-1}$ and $p_{i+1}$ are even, Proposition 4.5 tells us that $\mathcal{P}_{1}$ has the FAP with respect to its co- $(i-2)$-faces, and $\mathcal{P}_{2}$ has the FAP with respect to its 2 -faces. Then by Theorem 2.5 , there exists a regular polytope $\mathcal{P}$ with $(i+1)$-faces isomorphic to $\mathcal{P}_{1}$ and co- $(i-2)$-faces isomorphic to $\mathcal{P}_{2}$. Moreover, since $\mathcal{P}_{1}$ and $\mathcal{P}_{2}$ are both tight, Proposition 4.6 implies that $\mathcal{P}$ is also tight, and then since $\mathcal{P}$ is of type $\left\{p_{1}, \ldots, p_{n-1}\right\}$, we find that $|\Gamma(\mathcal{P})|=2 p_{1} \cdots p_{n-1}$. But also the $(i+1)$-faces of $\mathcal{P}$ are isomorphic to $\mathcal{P}_{1}$, and the co- $(i-2)$-faces are isomorphic to $\mathcal{P}_{2}$, and so the standard generators of $\Gamma(\mathcal{P})$ must satisfy all the relations of $\Gamma\left(p_{1}, \ldots, p_{n-1}\right)$. In particular, $\Gamma(\mathcal{P})$ is a quotient of $\Gamma\left(p_{1}, \ldots, p_{n-1}\right)$, and so $\left|\Gamma\left(p_{1}, \ldots, p_{n-1}\right)\right| \geq 2 p_{1} \cdots p_{n-1}$. On the other hand, $\left|\Gamma\left(p_{1}, \ldots, p_{n-1}\right)\right| \leq 2 p_{1} \cdots p_{n-1}$ by Lemma 4.4. Thus $\left|\Gamma\left(p_{1}, \ldots, p_{n-1}\right)\right|=$ $2 p_{1} \cdots p_{n-1}$, and hence also $\Gamma(\mathcal{P}) \cong \Gamma\left(p_{1}, \ldots, p_{n-1}\right)$, and $\mathcal{P} \cong \mathcal{P}\left(p_{1}, \ldots, p_{n-1}\right)$. Thus $\mathcal{P}\left(p_{1}, \ldots, p_{n-1}\right)$ is a tight polytope of type $\left\{p_{1}, \ldots, p_{n-1}\right\}$, and finally, since all the defining relations of $\Gamma\left(p_{1}, \ldots, p_{n-1}\right)$ have even length, we find that $\mathcal{P}\left(p_{1}, \ldots, p_{n-1}\right)$ is orientably-regular.

Note that the above theorem helps us deal with a large number of possibilities, once we have enough 'building blocks' in place. We are assuming that the $(n-1)$-tuple $\left(p_{1}, \ldots\right.$, $\left.p_{n-1}\right)$ is admissible, so that $p_{i-1}$ and $p_{i+1}$ are even divisors of $2 p_{i}$ whenever $p_{i}$ is odd. Now suppose that $n \geq 6$. If $p_{2}$ and $p_{4}$ are both even, then Theorem 4.7 will apply, and if not, then one of them is odd, say $p_{2}$, in which case $p_{1}$ and $p_{3}$ must both be even, and 
again Theorem 4.7 will apply. Hence this leaves us with just a few cases to verify, namely admissible $(n-1)$-tuples $\left(p_{1}, \ldots, p_{n-1}\right)$ with $n=4$ or 5 for which there is no $i$ such that $p_{i-1}$ and $p_{i+1}$ are both even.

The only such cases are as follows:

- $n=4$, with $p_{1}$ odd, $p_{2}$ even and $p_{3}$ even, or dually, $p_{1}$ even, $p_{2}$ even and $p_{3}$ odd,

- $n=4$, with $p_{1}$ odd, $p_{2}$ even and $p_{3}$ odd,

- $n=5$, with $p_{1}$ odd, $p_{2}$ even, $p_{3}$ even and $p_{4}$ odd.

We start with the cases where $n=4$ :

Proposition 4.8. If $p_{1}$ is odd, $p_{2}$ is an even divisor of $2 p_{1}$, and $p_{3} \geq 2$, then $\Gamma\left(p_{1}, p_{2}, p_{3}\right)$ is a string $C$-group, and $\mathcal{P}\left(p_{1}, p_{2}, p_{3}\right)$ is a tight orientably-regular polytope of type $\left\{p_{1}, q, p_{3}\right\}$, for some even $q$ dividing $p_{2}$.

Proof. Let $\Gamma=\Gamma\left(p_{1}, p_{2}, p_{3}\right)=\left\langle x_{0}, x_{1}, x_{2}, x_{3}\right\rangle$, and let $\bar{\Gamma}=\Gamma\left(p_{1}, 2, p_{3}\right)=\left\langle y_{0}, y_{1}, y_{2}\right.$, $\left.y_{3}\right\rangle$, where $y_{i}=\overline{x_{i}}$ is the image of $x_{i}$ under the natural epimorphism $\pi: \Gamma\left(p_{1}, p_{2}, p_{3}\right) \rightarrow$ $\Gamma\left(p_{1}, 2, p_{3}\right)$, for $0 \leq i \leq 3$. By Proposition 4.3, we know that $\Gamma\left(p_{1}, 2, p_{3}\right) \cong\left[p_{1}, 2, p_{3}\right]$. Also the subgroup $\left\langle x_{0}, x_{1}, x_{2}\right\rangle$ of $\Gamma$ covers $\left[p_{1}, 2\right]$, and this cover is one-to-one on $\left\langle x_{0}, x_{1}\right\rangle$, so $\left\langle x_{0}, x_{1}, x_{2}\right\rangle$ is a string C-group, by Proposition 2.1. A similar argument shows that $\left\langle x_{1}, x_{2}, x_{3}\right\rangle$ is a string C-group. Now the intersection of these two string C-groups is $\left\langle x_{1}, x_{2}\right\rangle$, since the intersection of their images in $\bar{\Gamma}$ is $\left\langle y_{0}, y_{1}, y_{2}\right\rangle \cap\left\langle y_{1}, y_{2}, y_{3}\right\rangle=\left\langle y_{1}, y_{2}\right\rangle$, and the kernel of $\pi$ is $\left\langle\left(x_{1} x_{2}\right)^{2}\right\rangle$. Hence by Proposition $2.2, \Gamma\left(p_{1}, p_{2}, p_{3}\right)$ is a string Cgroup, and the rest follows easily from Lemma 4.4.

Lemma 4.9. If $p_{i}=2$ for some $i$, then in $\Gamma\left(p_{1}, \ldots, p_{n-1}\right)=\left\langle x_{0}, \ldots, x_{n-1}\right\rangle$, we have $\left\langle x_{0}, \ldots, x_{i}\right\rangle \cong \Gamma\left(p_{1}, \ldots, p_{i}\right)$ and $\left\langle x_{i-1}, \ldots, x_{n-1}\right\rangle \cong \Gamma\left(p_{i}, \ldots, p_{n-1}\right)$.

Proof. First consider $\Lambda=\left\langle x_{0}, \ldots, x_{i}\right\rangle$. This is obtainable as a quotient of $\Gamma\left(p_{1}, \ldots, p_{i}\right)$ by adding extra relations. But also it can be obtained from the group $\Gamma\left(p_{1}, \ldots, p_{n-1}\right)$ by killing the unwanted generators $x_{i+1}, \ldots, x_{n-1}$. (Note that for $i+1 \leq j \leq n-1$, the relation $r_{j}=1$ and all relations of the form $\left(\rho_{j} \rho_{k}\right)^{m}=1$ become redundant and may be removed, and the same holds for the relations $r_{i}=1$ and $r_{i-1}=1$ since the assumption that $p_{i}=2$ implies that $\left[x_{i-1}, x_{i}\right]=\left(x_{i-1}, x_{i}\right)^{2}=1$ and hence that $x_{0}, \ldots, x_{i-1}$ commute with $x_{i}, \ldots, x_{n-1}$.) It follows that $\Lambda \cong \Gamma\left(p_{1}, \ldots, p_{i}\right)$. Also $\left\langle x_{i-1}, \ldots, x_{n-1}\right\rangle \cong$ $\Gamma\left(p_{i}, \ldots, p_{n-1}\right)$, by the dual argument.

Theorem 4.10. If $p_{1}$ is odd, $p_{2}$ is an even divisor of $2 p_{1}$, and $p_{3}$ is even, then $\mathcal{P}\left(p_{1}, p_{2}, p_{3}\right)$ is a tight orientably-regular polytope of type $\left\{p_{1}, p_{2}, p_{3}\right\}$.

Proof. First, the group $\Gamma\left(p_{1}, p_{2}, p_{3}\right)=\left\langle x_{0}, x_{1}, x_{2}, x_{3}\right\rangle$ covers $\Gamma\left(p_{1}, p_{2}, 2\right)=\left\langle y_{0}, y_{1}, y_{2}\right.$, $\left.y_{3}\right\rangle$, say, since $p_{3}$ is even. Also by Lemma 4.9 we know that $\left\langle y_{0}, y_{1}, y_{2}\right\rangle$ is isomorphic to $\Gamma\left(p_{1}, p_{2}\right)$, and hence in particular, $y_{1} y_{2}$ has order $p_{2}$. It follows that the order of $x_{1} x_{2}$ is also $p_{2}$, and then the conclusion follows from Proposition 4.8.

Theorem 4.11. If $p_{1}$ and $p_{3}$ are odd, and $p_{2}$ is an even divisor of both $2 p_{1}$ and $2 p_{3}$, then $\mathcal{P}\left(p_{1}, p_{2}, p_{3}\right)$ is a tight orientably-regular polytope of type $\left\{p_{1}, p_{2}, p_{3}\right\}$.

Proof. Under the given assumptions, the group $\Gamma\left(p_{1}, p_{2}, p_{3}\right)$ is obtained from the string Coxeter group $\left[p_{1}, p_{2}, p_{3}\right]=\left\langle x_{0}, x_{1}, x_{2}, x_{3}\right\rangle$ by adding the two extra relations $\left(x_{0} x_{1} x_{2} x_{1} x_{2}\right)^{2}=1$ and $\left(x_{3} x_{2} x_{1} x_{2} x_{1}\right)^{2}=1$. These imply that the element $\omega=\left(x_{1} x_{2}\right)^{2}$ 
is inverted under conjugation by each of $x_{0}$ and $x_{3}$, and hence by all the $x_{i}$. Now let $y_{i}=x_{i-1} x_{i}$ for $1 \leq i \leq 3$. These elements generate the orientation-preserving subgroup $\Gamma^{+}\left(p_{1}, p_{2}, p_{3}\right)$, and they all centralise $\omega=y_{2}^{2}$. It follows that $\Gamma^{+}\left(p_{1}, p_{2}, p_{3}\right)$ has presentation

$$
\left\langle y_{1}, y_{2}, y_{3} \mid y_{1}^{p_{1}}, y_{2}^{p_{2}}, y_{3}^{p_{3}},\left(y_{1} y_{2}\right)^{2},\left(y_{2} y_{3}\right)^{2},\left(y_{1} y_{2} y_{3}\right)^{2},\left[y_{1}, y_{2}^{2}\right],\left[y_{3}, y_{2}^{2}\right]\right\rangle .
$$

We now exhibit a permutation representation of this group on the Cartesian product $\mathbb{Z}_{p_{1}} \times \mathbb{Z}_{p_{2}}$, by letting each $y_{i}$ induce the permutation $\pi_{i}$, where

$$
\begin{gathered}
(j, k)^{\pi_{2}}=(j, k+1) \text { for all }(j, k), \\
(j, k)^{\pi_{1}}=\left\{\begin{array}{ll}
(j+1, k) & \text { for } k \text { even } \\
(j-1, k-2) & \text { for } k \text { odd, }
\end{array} \quad(j, k)^{\pi_{3}}= \begin{cases}(j, k-2 j) & \text { for } k \text { even } \\
(j, k+2(j-1)) & \text { for } k \text { odd } .\end{cases} \right.
\end{gathered}
$$

It is easy to see that $\pi_{1}$ and $\pi_{2}$ have orders $p_{1}$ and $p_{2}$ respectively (since $p_{2}$ divides $2 p_{1}$ ), and that the order of $\pi_{3}$ divides $p_{2} / 2$ and hence divides $p_{3}$. It is also easy to verify that they satisfy the other defining relations for $\Gamma^{+}\left(p_{1}, p_{2}, p_{3}\right)$, and thus we do have a permutation representation.

In particular, since $\pi_{2}$ has order $p_{2}$, so does $y_{2}=x_{1} x_{2}$, and again the conclusion follows from Proposition 4.8.

We now handle the remaining case.

Theorem 4.12. If $p_{1}$ and $p_{4}$ are odd, $p_{2}$ is an even divisor of $2 p_{1}$, and $p_{3}$ is an even divisor of $2 p_{4}$, then $\mathcal{P}\left(p_{1}, p_{2}, p_{3}, p_{4}\right)$ is a tight orientably-regular polytope of type $\left\{p_{1}, \ldots, p_{4}\right\}$.

Proof. Take $\Gamma=\Gamma\left(p_{1}, \ldots, p_{4}\right)=\left\langle x_{0}, \ldots, x_{4}\right\rangle$, and $\Lambda=\Gamma\left(p_{1}, p_{2}, 2, p_{4}\right)=\left\langle y_{0}, \ldots, y_{4}\right\rangle$. Then $\Gamma$ covers $\Lambda$, and this induces a cover from $\left\langle x_{0}, \ldots, x_{3}\right\rangle$ to $\left\langle y_{0}, \ldots, y_{3}\right\rangle$, which is isomorphic to $\Gamma\left(p_{1}, p_{2}, 2\right)$ by Lemma 4.9. Similarly we have a cover from $\left\langle x_{0}, x_{1}, x_{2}\right\rangle$ to $\left\langle y_{0}, y_{1}, y_{2}\right\rangle$, which is isomorphic to $\Gamma\left(p_{1}, p_{2}\right)$. But on the other hand, $\left\langle x_{0}, x_{1}, x_{2}\right\rangle$ is a quotient of $\Gamma\left(p_{1}, p_{2}\right)$, and hence these two groups are isomorphic. In particular, the cover from $\left\langle x_{0}, \ldots, x_{3}\right\rangle$ to $\Gamma\left(p_{1}, p_{2}, 2\right)$ is one-to-one on the facets, so $\left\langle x_{0}, \ldots, x_{3}\right\rangle$ is a string C-group. By a dual argument, $\left\langle x_{1}, \ldots, x_{4}\right\rangle$ is also a string C-group.

Next, let $\Delta=\Gamma\left(p_{1}, 2,2, p_{4}\right)=\left\langle z_{0}, \ldots, z_{4}\right\rangle$, and let $\pi$ be the covering homomorphism from $\Gamma$ to $\Delta$. The kernel of $\pi$ is the subgroup generated by $\left(x_{1} x_{2}\right)^{2}$ and $\left(x_{2} x_{3}\right)^{2}$, since the defining relations for $\Gamma=\Gamma\left(p_{1}, \ldots, p_{4}\right)$ imply that these two elements are centralised or inverted under conjugation by each generator $x_{i}$. In particular, ker $\pi \subseteq\left\langle x_{1}, x_{2}, x_{3}\right\rangle$. As also the intersection of $\left\langle z_{0}, \ldots, z_{3}\right\rangle$ and $\left\langle z_{1}, \ldots, z_{4}\right\rangle$ in $\Delta$ is $\left\langle z_{1}, z_{2}, z_{3}\right\rangle$, it follows that intersection of $\left\langle x_{0}, \ldots, x_{3}\right\rangle$ and $\left\langle x_{1}, \ldots, x_{4}\right\rangle$ is $\left\langle x_{1}, x_{2}, x_{3}\right\rangle$. Hence $\Gamma$ is a string C-group.

Now $\left\langle x_{0}, x_{1}, x_{2}\right\rangle \cong \Gamma\left(p_{1}, p_{2}\right)$, and by Theorem 3.1 we know the polytope $\mathcal{P}\left(p_{1}, p_{2}\right)$ has type $\left\{p_{1}, p_{2}\right\}$. Similarly $\left\langle x_{2}, x_{3}, x_{4}\right\rangle \cong \Gamma\left(p_{3}, p_{4}\right)$, and $\mathcal{P}\left(p_{3}, p_{4}\right)$ has type $\left\{p_{3}, p_{4}\right\}$. It follows that $\mathcal{P}\left(p_{1}, p_{2}, p_{3}, p_{4}\right)$ is an orientably-regular polytope of type $\left\{p_{1}, \ldots, p_{4}\right\}$. In particular, the order of $x_{i-1} x_{i}$ is $p_{i}$ (for $\left.1 \leq i \leq 4\right)$, and so by Lemma $4.4, \mathcal{P}\left(p_{1}, p_{2}, p_{3}, p_{4}\right)$ is tight.

This gives us all the building blocks we need. With the help of Theorem 4.7, we now know that $\mathcal{P}\left(p_{1}, \ldots, p_{n-1}\right)$ is a tight orientably-regular polytope of type $\left\{p_{1}, \ldots, p_{n-1}\right\}$ whenever $\left(p_{1}, \ldots, p_{n-1}\right)$ is admissible, and the proof of Theorem 1.1 is complete. 


\section{Tight non-orientably-regular polytopes}

We have not yet been able to completely characterise the Schläfli symbols of tight, nonorientably-regular polytopes, but we have made some partial progress. For example, we can easily find an infinite family of tight, non-orientably-regular polyhedra.

Theorem 5.1. For every odd positive integer $k$, there exists a non-orientably-regular tight polyhedron of type $\{3 k, 4\}$, with automorphism group $\Lambda(k)$ having presentation

$$
\left\langle\rho_{0}, \rho_{1}, \rho_{2} \mid \rho_{0}^{2}, \rho_{1}^{2}, \rho_{2}^{2},\left(\rho_{0} \rho_{1}\right)^{3 k},\left(\rho_{0} \rho_{2}\right)^{2},\left(\rho_{1} \rho_{2}\right)^{4}, \rho_{0} \rho_{1} \rho_{2} \rho_{1} \rho_{0} \rho_{1} \rho_{2} \rho_{1} \rho_{2}\right\rangle .
$$

Proof. We note that $\Lambda(1)$ is the automorphism group of the hemi-octahedron (of type $\{3,4\}$ ), and that $\Lambda(k)$ covers $\Lambda(1)$, for every $k$. Hence in each $\Lambda(k)$, the order of $\rho_{1} \rho_{2}$ is 4 (and not 1 or 2 ). Next, because the covering is one-to-one on the vertex-figures, it follows from Proposition 2.1 that $\Lambda(k)$ is a string C-group. Also $\Lambda(k)$ has a relation of odd length, and so it must be the automorphism group of a non-orientably-regular polyhedron.

Now let $N$ be the subgroup generated by the involutions $\rho_{2}$ and $\rho_{1} \rho_{2} \rho_{1}$. Since their product has order 2 , this is a Klein 4-group. Moreover, $N$ is normalised by $\rho_{1}$ and $\rho_{2}$, and also by $\rho_{0}$ since $\rho_{0}$ centralises $\rho_{2}$ and the final relation in the definition of $\Lambda(k)$ gives $\left(\rho_{1} \rho_{2} \rho_{1}\right)^{\rho_{0}}=\rho_{1} \rho_{2} \rho_{1} \rho_{2}$. It is now easy to see that $N$ is the normal closure of $\left\langle\rho_{2}\right\rangle$. The quotient $\Lambda(k) / N$ is isomorphic to $\left\langle\rho_{0}, \rho_{1} \mid \rho_{0}^{2}, \rho_{1}^{2},\left(\rho_{0} \rho_{1}\right)^{3 k}\right\rangle$, with the final relator for $\Lambda(k)$ becoming trivial, and so $\Lambda(k) / N$ is dihedral of order $6 k$. In particular, this shows that $\rho_{0} \rho_{1}$ has order $3 k$ (and that $|\Lambda(k)|=|\Lambda(k) / N||N|=24 k$ ).

The computational data that we have on polytopes with up to 2000 flags (obtained with the help of MAGMA [1]) suggests that these polyhedra are the only tight non-orientablyregular polyhedra of type $\{p, q\}$ with $p$ odd.

Using Theorem 5.1, it is possible to build tight, non-orientably-regular polytopes in much the same way as we did in Theorem 4.7. In particular, the regular polytope with automorphism group $\Lambda(k)$ has the FAP with respect to its 2-faces, and its dual has the FAP with respect to its vertex-figures (co-0-faces). Then by Theorem 2.5 , we know there are tight, non-orientably-regular polytopes of type $\{4,3 k, 4\}$ for each odd $k$, and of type $\{4,3 k, r\}$ for each odd $k$ and each even $r$ dividing $3 k$. It is possible to continue in this fashion, building tight non-orientably-regular polytopes of every rank.

Finally, just as with orientably-regular polytopes, there are some kinds of Schläfli symbol for which no examples can be constructed using Theorem 2.5. In fact (and in contrast with the situation for orientably-regular polytopes), there seem to be no tight nonorientably-regular polytopes of some of these types at all. For example, there are no tight regular polytopes of type $\{3,4, r\}$ with $r \geq 3$ and with 2000 flags or fewer, but the reason for this is not clear.

\section{References}

[1] W. Bosma, J. Cannon and C. Playoust, The Magma algebra system I, The user language, J. Symbolic Comput. 24 (1997), 235-265.

[2] M. Conder, Lists of all regular polytopes with up to 2000 flags, http://www math . auckland.ac.nz/ conder.

[3] M. Conder, The smallest regular polytopes of any given rank, Adv. Math. 236 (2013), 92-110. 
[4] M. D. E. Conder and T. W. Tucker, Regular Cayley maps for cyclic groups, Trans. Amer. Math. Soc. 366 (2014), 3585-3609.

[5] G. Cunningham, Minimal equivelar polytopes, Ars Math. Contemp. 7 (2014), 299-315.

[6] M. I. Hartley, An atlas of small regular abstract polytopes, Period. Math. Hung. 53 (2006), 149-156.

[7] I. Hubard, Two-orbit polyhedra from groups, Eur. J. Comb. 31 (2010), 943-960.

[8] P. McMullen and E. Schulte, Abstract regular polytopes, Encyclopedia of Mathematics and its Applications, vol. 92, Cambridge University Press, Cambridge, 2002.

[9] E. Schulte, Amalgamation of regular incidence-polytopes, Proc. London Math. Soc. 3 (1988), 303-328.

[10] E. Schulte and A. Ivić Weiss, Chiral polytopes, Applied geometry and discrete mathematics, DIMACS Ser. Discrete Math. Theoret. Comput. Sci., vol. 4, Amer. Math. Soc., Providence, RI, 1991, pp. 493-516. 